Wie funktioniert es?

Nephrologe 2019 · 14:475-478

https://doi.org/10.1007/s11560-019-0363-6

Online publiziert: 8. August 2019

(c) Der/die Autor(en) 2019

\section{Redaktion}

F. Schweda, Regensburg

\author{
J. Voelk| ${ }^{1,2,3}$ \\ ${ }^{1}$ Institut für Physiologie and Pathophysiologie, Johannes Kepler Universität Linz, Linz, Österreich \\ ${ }^{2}$ Medizinische Klinik mit Schwerpunkt Nephrologie und Internistische Intensivmedizin, Charité- \\ Universitätsmedizin Berlin, Berlin, Deutschland \\ ${ }^{3}$ Deutsches Zentrum für Herz-Kreislauf-Forschung Berlin, Berlin, Deutschland
}

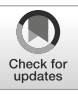

\title{
Welche Rolle spielen Phosphathaushalt und Gefäßverkalkungen bei der chronischen Niereninsuffizienz?
}

\section{Einleitung}

Die aktuelle Literatur weist relativ eindeutig darauf hin, dass Phosphat nachteilige Effekte auf das kardiovaskuläre System verursachen kann. Mit unseren aktuellen Ernährungsgewohnheiten nehmen wir (zu-)viel Phosphat auf (ca. 1000-1500 mg/Tag). Unser Körper muss den Phosphathaushalt eng kontrollieren. Eine Dysregulation der Phosphatbalance tritt besonders bei chronischer Niereninsuffizienz auf. Dabei werden die kardiovaskulären Effekte der Phosphattoxizität wahrscheinlich stark beschleunigt und führen zu weitreichenden Gefäßverkalkungen [1].

\section{Phosphathomöostase}

Das mit der Nahrung aufgenommene Phosphat wird besonders im Dünndarm resorbiert, zu etwa gleichen Teilen durch transzellulären aktiven Natrium-Phosphat-Kotransport (NaPi-IIb) und durch parazellulären Transport. In der Nahrung künstlich zugesetzte Phosphate sind wahrscheinlich viel besser resorbierbar als in pflanzlicher Nahrung vorhandene Phosphate. Die Phosphatspiegel im Blut zeigen eine deutliche zirkadiane Rhythmik und sind vormittags gering, aber nachts hoch [1-3].

Bei physiologischem pH-Wert würde das zirkulierende Phosphat eigentlich mit Kalzium komplexieren und Hydroxylapatitkristalle bilden. Dies wird im Blut und Gefäßgewebe durch sog. Kalzifizierungsinhibitoren, wie Fetuin-A, MatrixGLA ( $\gamma$-Carboxyglutaminsäure)-Protein oder Pyrophosphat verhindert. Diese Kalzifizierungsinhibitoren können das Wachstum von Hydroxylapatit verhindern, indem sie Kalziumphosphatpartikel in primären Kalzium-PhosphatProteinpartikeln (CPP) umhüllen. Wenn der Mineralisierungsstress zu groß wird oder zu wenig Kalzifizierungsinhibitoren vorhanden sind, bilden sich „versagende" sekundäre CPP, die eine ektope Kalzifizierung fördern. Die Eigenschaften der Balance zwischen Phosphat und „Anti-Kalzifizierung“ lassen sich durch einen Test der Kalzifizierungsneigung im Patientenblut bestimmen [4].

Unter physiologischen Bedingungen kann Phosphat mit Kalzium in den Knochen eingelagert werden. Das zirkulierende Phosphat wird auch in der Niere filtriert und v.a. im proximalen Tubulus durch die Natrium-PhosphatKotransporter NaPi-IIa, NaPi-IIc und PiT-2 transzellulär rückresorbiert. Die zirkulierenden Phosphatspiegel werden durch 3 Hormone reguliert [5]:

- Parathormon (PTH) steuert die frühe Phase der Phosphatrückresorption nach Phosphataufnahme, und fördert die Freisetzung von FGF23 („fibro-

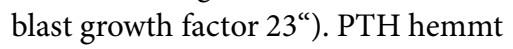
die Phosphatrückresorption durch Internalisierung der renalen Phosphattransporter. PTH fördert auch die Freisetzung von Kalzium und
Phosphat aus dem Knochen und die Bildung von Calcitriol in der Niere.

- FGF23 wird im Knochen gebildet und führt wie PTH zur vermehrten Phosphatausscheidung in der Niere. FGF23 hemmt aber die Bildung von Calcitriol in der Niere und die PTHFreisetzung in der Nebenschilddrüse.

- Aktives Vitamin D3 $\left(1,25[\mathrm{OH}]_{2} \mathrm{D}_{3}\right.$ oder Calcitriol) wird im proximalen Tubulus der Niere gebildet. Calcitriol fördert die Aufnahme von Kalzium und Phosphat im Darm durch Steigerung des aktiven Transports. Ob Calcitriol auch die renale Phosphatreabsorption steigern kann, ist noch umstritten. Calcitriol hemmt die PTH-Freisetzung und fördert die FGF23-Freisetzung.

Kurz. Es existiert ein engmaschiger Regelkreis, der die Phosphatspiegel in einem konstanten Rahmen hält. Kalzifizierungsinhibitoren verhindern die Präzipitation von zirkulierendem Kalzium und Phosphat in Blut und Gewebe (• Abb. 1).

\section{Phosphatdysregulation bei chronischer Niereninsuffizienz}

Bei abnehmender Nierenfunktion nimmt die Filtration von Phosphat ab, wodurch das endokrine System zur Phosphatausscheidung aktiviert wird. Bereits bei einer Reduktion der glomerulären Filtrationsrate (GFR) auf $60 \mathrm{ml} / \mathrm{min} / 1,73 \mathrm{~m}^{2}$ steigen die FGF23-Spiegel an, und die Calcitriol- 


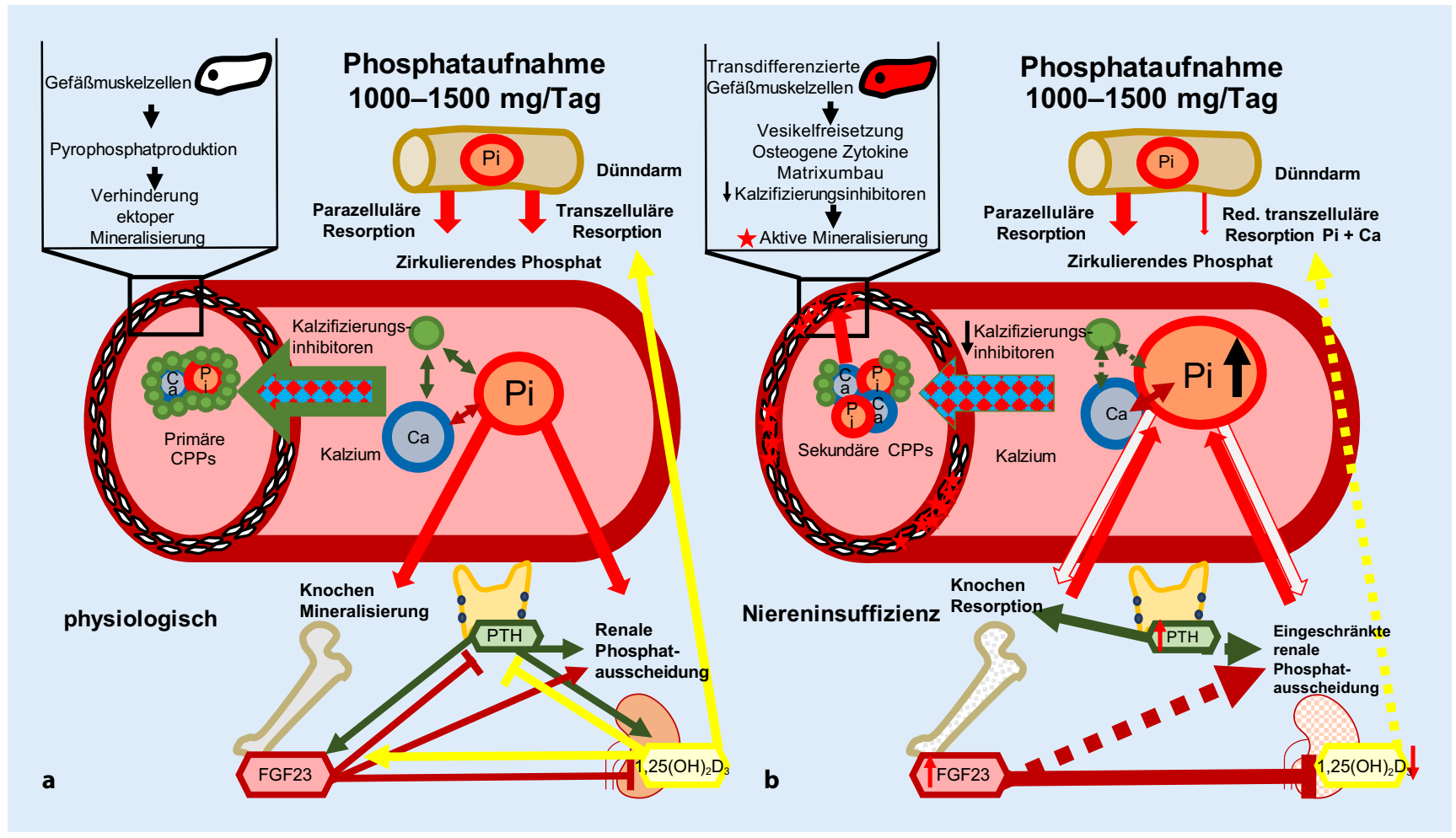

Abb. 1 ॥ Vermutete Interaktion von Phosphat(Pi)-Haushalt und medialer Gefäßverkalkung unter physiologischen Bedingungen (a) und bei fortgeschrittener Niereninsuffizienz (b). Ca Kalzium, CPP Kalzium-Phosphat-Proteinpartikel, PTH Parathormon, FGF23 "fibroblast growth factor 23". (Mit freundlicher Genehmigung, @ J. Völkl. Alle Rechte vorbehalten)

spiegel sinken. Die PTH-Spiegel steigen erst verzögert bei weiter abnehmender Nierenfunktion an. Dadurch wird die renale Phosphatreabsorption reduziert, und die Phosphatausscheidung bleibt trotz reduzierter Filtration konstant [1].

Wenn die GFR auf unter $30 \mathrm{ml} / \mathrm{min} / 1,73 \mathrm{~m}^{2}$ abfällt, kann die reduzierte Phosphatfiltration nicht mehr durch Hemmung der renalen Reabsorption kompensiert werden. Die Phosphatspiegel steigen an, und es kommt zu einer Hyperphosphatämie. Dadurch wird die Freisetzung von FGF23 im Knochen weiter stimuliert, und in Folge werden die Calcitriolspiegel weiter reduziert. Interessanterweise kommt es in der Niere zur Reduktion von $\alpha$-Klotho, dem Korezeptor für FGF23 in der Niere, wodurch die phosphaturischen Effekte von FGF23 geschwächt werden könnten [1].

Die reduzierten Calcitriolspiegel führen zu einer geringeren transzellulären Aufnahme von Kalzium und Phosphat im Darm. Ob und wie die parazelluläre Phosphatabsorption reguliert wird, ist unklar. Bei einer hohen Phosphatauf- nahme könnte zumindest theoretisch die Hemmung des aktiven Phosphattransports aber völlig von parazellulärem Phosphattransport kompensiert werden. Eventuell kann der parazelluläre Phosphattransport durch neue Medikamente wie den NHE3(,sodium-hydrogen exchanger 3“)-Inhibitor Tenapanor reduziert werden [2].

Die reduzierten Calcitriolspiegel führen zu einer abnehmenden intestinalen Kalziumresorption. Bei Versagen oder Überlastung der Kalzifizierungsinhibitoren kommt es zur Komplexierung von Kalzium mit Phosphat. Durch diese Effekte wird der „calcium-sensing receptor" an der Nebenschilddrüse nicht mehr ausreichend aktiviert, was zur vermehrten PTH-Freisetzung führt, wodurch vermehrt Kalzium und Phosphat aus dem Knochen resorbiert wird [5].

Merke. Bei fortgeschrittener chronischer Niereninsuffizienz wird Phosphat trotz erhöhter FGF23- und PTH-Spiegel nicht mehr ausreichend renal ausgeschieden. Die intestinale Aufnahme von Phosphat kann wahrscheinlich nicht kompensie- rend reduziert werden. Phosphat wird mit Kalzium vermehrt aus dem Knochen freigesetzt. Die hohen Phosphatspiegel überlasten die Kalzifizierungsinhibitoren (• Abb. 1).

\section{Phosphatinduzierte Gefäßverkalkung}

Es ist mittlerweile sehr gut belegt, dass Hyperphosphatämie bei Niereninsuffizienz mit schädlichen Effekten am kardiovaskulären System und mit erhöhter kardiovaskulärer Mortalität assoziiert ist. Interessanterweise zeigen sich ähnliche Assoziationen auch in der gesunden Bevölkerung, d.h. auch bei Phosphatspiegeln, die sich im „Normbereich“ befinden. Durch welche Mechanismen Phosphat nachteilige Effekte vermittelt, ist noch nicht endgültig belegt. Ein wichtiger Effekt könnte aber die Entstehung einer medialen Gefäßverkalkung (Mönckeberg-Mediasklerose) sein, die in der Muskelschicht der Arterien auftritt. Diese unterscheidet sich deutlich von der intimalen Verkalkung der atherosklerotischen Plaque $[1,6]$. 
Bei der medialen Gefäßverkalkung spielen die glatten Gefäßmuskelzellen wahrscheinlich eine zentrale Rolle. Unter normalen Bedingungen verhindern diese Zellen eine ektope Mineralisierung durch Produktion von Kalzifizierungsinhibitoren wie Pyrophosphat. Diese Zellen können sich aber unter hohen Phosphatspiegeln in Zellen mit osteoblastischen und chondroblastischen Eigenschaften umwandeln. Diese Transdifferenzierung kann auch durch entzündliche Prozesse und Hyperglykämie gefördert werden [7].

Die transdifferenzierten Gefäßmuskelzellen verursachen mehrere Effekte [7]:

- Sie verhindern die Funktion der Kalzifizierungsinhibitoren, v. a. indem sie den Kristallisationsinhibitor Pyrophosphat spalten.

- Die transdifferenzierten Gefäßmuskelzellen setzen apoptotische und exosomale Vesikel frei, die als Kalzifizierungsnidus die Bildung von Hydroxyapatitkristallen steuern.

- Die physiologische extrazelluläre Matrix wird abgebaut und in eine knochenähnliche Matrix umgewandelt.

- Es werden osteogene und inflammatorische Zytokine freigesetzt, die die Umwandlung weiterer Gefäßmuskelzellen verstärken.

Durch diese Effekte kommt es zur Entstehung eines prokalzifizierenden Milieus in den Arterien, das gewisse Parallelen zur Knochenmineralisierung aufweist. Dadurch wird eine aktive und regulierte Mineralisierung der Gefäße ermöglicht. Wie sich diese Verkalkungen nachteilig auf das kardiovaskuläre System auswirken, ist nicht genau geklärt. Wahrscheinlich spielt dabei die Versteifung der Gefäße mit vermehrter kardialer Belastung und Störung der Autoregulation der Organdurchblutung eine bedeutende Rolle $[6,8]$.

Merke. Hyperphosphatämie bei chronischer Nierenerkrankung fördert die Entstehung von Gefäßverkalkungen und osteo-/chondrogene Transdifferenzierung von Gefäßmuskelzellen. Die transdifferenzierten Gefäßmuskelzellen leiten eine aktive Mineralisierung der Gefäße ein.

\section{Fazit für die Praxis}

- Der Phosphathaushalt ist wahrscheinlich von immer noch unterschätzter Bedeutung, und es gibt noch zahlreiche Lücken im Verständnis bei Niereninsuffizienz.

- Diätetische Maßnahmen zur Reduktion von leicht resorbierbaren Phosphaten und die Gabe von Phosphatbindern erscheinen sinnvoll.

- Eine Hemmung der intestinalen Phosphataufnahme ist aber wahrscheinlich nicht ausreichend, um Phosphatüberladung zu kompensieren, da Phosphat auch aus dem Knochen freigesetzt wird.

- In der präklinischen Forschung gibt es erste Ansätze, wie die toxischen Effekte von Phosphat und die Entstehung von Gefäßverkalkungen reduziert werden könnten. Zu diesen Ansätzen zählen beispielsweise Magnesium- oder Zinksupplementation, Aldosteronblockade oder VitaminK-Behandlung, bislang gibt es aber keine klinischen Daten zur breiten Anwendung derartiger Ansätze.

- Neue diagnostische Tests wie die Kalzifizierungsneigung im Blut könnten in Zukunft wertvolle klinische Erkenntnisse liefern.

\section{Korrespondenzadresse}

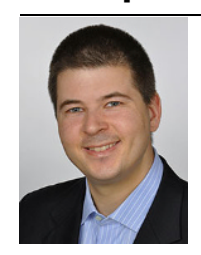

Univ.-Prof. Dr. J. Voelk

Institut für Physiologie and Pathophysiologie, Johannes Kepler Universität Linz Altenberger Straße 69, 4040 Linz, Österreich jakob.voelkl@jku.at

Funding. Open access funding provided by Johannes Kepler University Linz.

Interessenkonflikt. J. Voelkl gibt an, dass kein Interessenkonflikt besteht.

Open Access. Dieser Artikel wird unter der Creative Commons Namensnennung 4.0 International Lizenz (http://creativecommons.org/licenses/by/4.0/deed. de) veröffentlicht, welche die Nutzung, Vervielfältigung, Bearbeitung, Verbreitung und Wiedergabe in jeglichem Medium und Format erlaubt, sofern Sie den/die ursprünglichen Autor(en) und die Quelle ordnungsgemäßnennen, einen Linkzur Creative Com- mons Lizenz beifügen und angeben, ob Änderungen vorgenommen wurden.

\section{Literatur}

1. Vervloet MG et al (2017) The role of phosphate in kidney disease. Nat Rev Nephrol 13(1):27-38

2. Levi M et al (2019) Mechanisms of phosphate transport. Nat Rev Nephrol 15(8):482-500

3. St-JulesDEetal (2017) Examining the proportion of dietary phosphorus from plants, animals, and food additives excreted in urine. J Ren Nutr 27(2):78-83

4. Pasch A, Jahnen-Dechent W, Smith ER (2018) Phosphate, calcification in blood, and mineral stress: the physiologic blood mineral buffering system and its association with cardiovascular risk. Int JNephrol 2018:9182078

5. Jacquillet G, Unwin RJ (2019) Physiological regulation of phosphate by vitamin $D$, parathyroid hormone (PTH) and phosphate (pi). Pflugers Arch 471(1):83-98

6. VoelkI J, Cejka D, Alesutan I (2019a) An overview of the mechanisms in vascular calcification during chronic kidney disease. Curr Opin Nephrol Hypertens 28(4):289-296

7. Voelkl J et al (2019b) Signaling pathways involved in vascular smooth muscle cell calcification during hyperphosphatemia. Cell Mol Life Sci. https://doi. org/10.1007/s00018-019-03054-z

8. Lanzer P et al (2014) Medial vascular calcification revisited: review and perspectives. Eur Heart J 35(23):1515-1525 
Hier steht eine Anzeige.

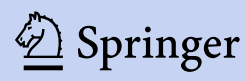

